support, Hannah Rowe Grant/research support from: Novartis UK Investigator Initiated non-clinical research funding support, Peter Millner: None declared, Peter Loughenbury: None declared, Abhay S Rao: None declared, Robert Dunsmuir: None declared, Charlie Bridgewood: None declared, Yasser El-Sherbiny: None declared, Dennis McGonagle Grant/research support from: Janssen Research \& Development, LLC

DOI: 10.1136/annrheumdis-2020-eular.5262

\title{
THU0029 IDENTIFICATION AND COMPARING OF HUB GENES IN SYSTEMIC LUPUS ERYTHEMATOSUST AND B CELLS BY BIOINFORMATIC ANALYSIS
}

Q. Zhou ${ }^{1}$, L. Long ${ }^{1} .{ }^{1}$ Sichuan Provincial People's Hospital, Department of Rheumatology and Immunology, Chengdu, China

Background: Systemic lupus erythematosus (SLE) is a chronic autoimmune disorder that can virtually involve any organ system of the body. Many efforts have been made to elucidate the pathogeny, but the molecular mechanisms are still not understood. Lymphocytes are considered to play an important role in SLE pathogenesis. Aberrantly activated $T$ cells mediate inflammatory responses and activate $B$ cells to differentiate and produce autoantibodies, resulting in multisystem manifestations $[1,2]$. With the wide use of gene technique, more genetic studies on SLE were performed and differentially expressed genes (DEGs) were identified. Integrating and re-analyzing these data can help us understand the molecular mechanisms and identify diagnostic and therapeutic targets of SLE.

Objectives: In this study, we downloaded the microarray datasets GSE4588 and GSE10325 from Gene Expression Omnibus (GEO) database to identify the candidate genes in $T$ and $B$ cells respectively.

Methods: Datasets GSE4588 and GSE10325 were downloaded from GEO (http://www.ncbi.nlm.nih.gov/geo). The DEGs between T or B cells and control samples were screened using GEO2R (http://www.ncbi.nlm.nih.gov/geo/ geo2r). logFC Ifold changel $>1$ and $P$-value $<0.05$ were considered statistically significant. To analyze the function of DEGs, biological analyses were performed using DAVID database (http://david.ncifcrf.gov). $\mathrm{P}<0.05$ was considered statistically significant. The PPI networks of DEGs were constructed using STRING database, and an interaction with a combined score $>0.4$ was considered statistically significant. The PPI networks were drawn using Cytoscape and the most significant module was identified using MCODE. The criteria for selection were: MCODE scores $>5$, degree cut-off $=2$, node score cut-off=0.2, Max depth=100 and k-score=2. The hub genes were selected with degrees $\geq 10$.

Results: After standardization of the microarray results, DEGs in T and B cells were identified respectively (Fig. 1)

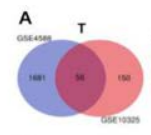

C
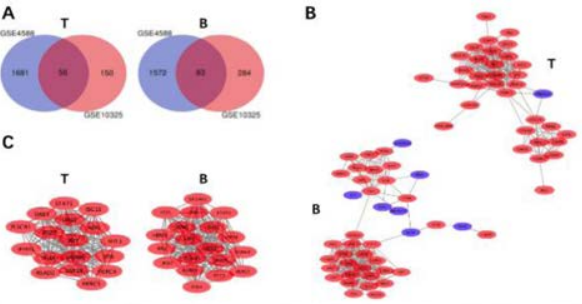

Figure 1. Venn diagram, PPI network and the most significant module of DEGs in SLE. (A) The overlap between 2 datasets contained 56 genes in $T$ cells and 83 in B cells. (B) PPI networks were constructed using Cytoscape, (C) The most significant module in $T$ cells had 17 nodes and 193 edges, while that in B cells had 19 nodes and 171 edges. Upregulated genes are in red; downregulated genes are in blue.

Changes in biological processes in $\mathrm{T}$ and $\mathrm{B}$ cells were both mainly enriched in type I interferon signalling pathway, defense response to virus, and negative regulation of viral genome replication. Changes in cell component in T cells was enriched in the cytosol while in B cells it was in cytoplasm. KEGG pathway analysis revealed that the DEGs of $T$ cells were mainly enriched in influenza $A$, measles, herpes simplex infection and hepatitis $C$, while DEGs of $B$ cells were mainly enriched in measles. Changes in molecular function were not listed because the $\mathrm{p}$ values were $\geq 0.05$.

4 genes were identified as hub genes ( 2 in each cell population). In T cells, the hub genes are PLSCR1 and GINS2. PLSCR1 may contribute to the prothrombotic tendency in SLE. GINS2 is involved in the initiation of DNA replication and cell cycle progression. In B cells, the hub genes are ISG15 and TOP2A. Increased ISG15 is correlated with lymphocytopenia in SLE patients. TOP2A encodes a DNA topoisomerase and anti-topoisomerase II antibody could be found in SLE. Conclusion: In our study, 2 mRNA microarray datasets were analyzed to obtain DEGs between SLE T and B cells versus healthy controls. A total of 56 DEGs were identified in T cells and 83 in B cells. Most of the DEGs were upregulated. Changes in biological processes in $T$ and $B$ cells were mainly related to type I interferon signalling pathway and anti-virus function. KEGG also showed the same. PLSCR1 and GINS2 were hub genes in T cells while ISG15 and TOP2A were hub genes in B cells. Overexpression of these genes might play an important role in the pathogenesis of SLE.

\section{References:}

[1] Ohl, K. and K. Tenbrock, Regulatory T cells in systemic lupus erythematosus. Eur J Immunol, 2015. 45(2): p. 344-55.

[2] Moulton, V.R. and G.C. Tsokos, $T$ cell signaling abnormalities contribute to aberrant immune cell function and autoimmunity. J Clin Invest, 2015. 125(6): p. 2220-7.

Disclosure of Interests: None declared

DOI: 10.1136/annrheumdis-2020-eular.2525

\section{THURSDAY, 04 JUNE 2020}

\section{Adaptive immunity ( $T$ cells and $B$ cells) in rheumatic diseases}

\section{THU0030 DISTINCT EFFECTS OF FIVE JAK INHIBITORS IN THE MODULATION OF HUMAN B CELL ACTIVATION}

N. Frede ${ }^{1}$, J. Hueppe ${ }^{1}$, R. Lorenzetti ${ }^{1}$, A. Troilo ${ }^{1}$, M. T. Schleyer ${ }^{1}$, R. Voll ${ }^{1}$, J. Thiel $^{1}$, N. Venhoff ${ }^{1}$, M. Rizzi ${ }^{1}{ }^{1}$ Medical Center - University of Freiburg, Faculty of Medicine, University of Freiburg, Freiburg im Breisgau, Germany

Background: JAK inhibitors have been successfully introduced in the treatment of rheumatoid arthritis (RA) and psoriatic arthritis and are in clinical trials for numerous other autoimmune diseases. JAK inhibition effectively reduces cytokine-mediated activation and survival of pathology-driving immune cells by targeting signaling downstream of cytokine receptors. The outcome of such immunomodulation hence will largely depend on the intrinsic expression of the four different JAKs, the cytokine environment and the targeted cell type. Comparative studies investigating the effect on B cells are lacking. In light of the use of JAK inhibitor treatment in autoantibody mediated diseases, the study of the B cell compartment represents a milestone to assess their potential.

Objectives: We thus aimed to study the B cell compartment as well as B cell function under JAK inhibition in RA patients and to compare the specific effect the JAK inhibitors tofacitinib (pan-JAK), baricitinib (JAK1/2), ruxolitinib (JAK1/2), upadacitinib and filgotinib (selective JAK1) on in vitro B cell activation, differentiation, proliferation, and class switch.

Methods: B cell subpopulations in RA patients treated with baricitinib or tofacitinib was assessed by flow cytometric analysis of peripheral blood mononuclear cells. For in vitro studies, magnetically isolated total B cells from healthy donors were stimulated T-cell -independently with $\mathrm{CpG}$ and treated with scalar doses of the JAK inhibitors tofacitinib, baricitinib, ruxolitinib, upadacitinib and filgotinib. Flow cytometric analysis was performed on days 0,3 and 6 . Cytokine secretion was measured by Cytokine Multiplex Assay.

Results: B cell phenotyping of RA patients treated with JAK inhibitors baricitinib or tofacitinib showed an increase in marginal zone (MZ) B cells. To investigate this further, we turned to an in vitro model of T-cell-independent B cell activation with CpG via TLR9, known to support MZ B cell expansion Here, JAK1/2 and selective JAK1 inhibitor treatment led to a dose-dependent decrease of total B cell numbers. When assessing B cell-subpopulations, we observed an altered B cell differentiation with a significant increase in MZ-like $B$ cells under JAK inhibition, which led to a subsequent increase in plasmablast differentiation in the first days. This effect was more pronounced upon pan-JAK inhibitor treatment than JAK1 or JAK1/2 inhibition, indicating that broader JAK inhibition is associated with a stronger effect (tofa > ruxo $>$ bari $>$ upa $>$ filgo).

Notably, we further detected a significant dose-dependent reduction of switched memory formation, strongest with JAK $1 / 2$ inhibition (upa > ruxo $>$ bari $>$ tofa $>$ filgo). Consistent with this finding, we observed decreased AID expression under JAK inhibition. Concomitantly, induction of STAT3 expression and STAT3 phosphorylation were reduced under JAK inhibition, suggesting that downstream signalling was abrogated.

To assess the role of autocrine signaling in this system, we measured cytokine secretion upon JAK inhibition and found that JAK2 inhibition led to reduced IL10 secretion. This in turn resulted in an increase of inflammatory cytokines such as IL6, TNF, highlighting the importance of B cell as cytokine-secreting cell type.

Conclusion: In a T-independent in vitro B cell model JAK inhibition led to a reduced total $B$ cell number as well as reduced switched memory development whereas MZ-like B cells were increased. Especially JAK2 inhibition strongly impaired switched memory formation. JAK inhibition does not only impact cytokine signalling but also leads to changes in cytokine secretion dynamics and amounts, potentially impacting other cell types.

In conclusion, JAK inhibition has a major effect on B cell activation and maturation, with differential outcomes between JAK inhibitors hinting towards distinct and unique effects on $B$ cell homeostasis. 
Disclosure of Interests: None declared

DOI: 10.1136/annrheumdis-2020-eular.5763

\section{THU0031 \\ ABATACEPT ALTERS THE FREQUENCY OF IMMUNOREGULATORY AND EFFECTOR T CELL SUBPOPULATIONS IN RHEUMATOID ARTHRITIS}

B. Dreo $^{1}$, B. Prietl ${ }^{2,3}$, S. Kofler ${ }^{3}$, H. Sourij ${ }^{2,3}$, A. Lackner ${ }^{1}$, F. Moazedi-Fürst ${ }^{1}$, M. D'orazio ${ }^{1}$, M. Stradner ${ }^{1}$, W. Graninger ${ }^{1}$, H. P. Brezinschek ${ }^{1}{ }^{1}$ Medical University of Graz, Division of Rheumatology and Immunology, Graz, Austria; ${ }^{2} \mathrm{CBmed} \mathrm{GmbH}$ - Center for Biomarker Research in Medicine, Graz, Austria; ${ }^{3}$ Medical University of Graz, Division of Endocrinology and Diabetology, Graz, Austria

Background: Under physiological conditions, $\mathrm{T}$ regulatory cells (Tregs) are responsible for the downregulation of the immune response. In autoimmune diseases, such as rheumatoid arthritis (RA), auto-inflammation is driven by an imbalance of activation and downregulation of immunological pathways. Thus, treatment plans for autoimmune diseases often involve the enhancement of immunoregulatory pathways by administering inhibitors of costimulation, i.e. CTLA-4-Ig (abatacept, ABA). ABA binds specifically to CD80 and CD86 on antigen presenting cells (APC). Consequently, T cell activation via the CD28 receptor is blocked. Previous studies have demonstrated surprising effects of abatacept on Tregs, specifically decreased frequency of these cells but enhancement in their function ${ }^{1}$. Whether these alterations can only be found in patients with ABA treatment, or whether they are also present in patients receiving other anti-inflammatory drugs is currently unknown.

Objectives: The aim of our research was to delineate the impact of ABA on the different subsets of effector and regulatory T cells in RA and compare these findings with patients receiving tocilizumab (TCZ) or rituximab (RTX).

Methods: Peripheral blood samples from 56 RA patients (median \pm SE; age: $60.5 \pm 1.3$ years, female ratio: 0.7 , disease duration: $17.9 \pm 2.1$ years; respectively) were drawn over a sampling period of 2 years. Freshly isolated PBMCs of RA patients were stained with fluorochrome-labelled antibodies and $T$ cell subsets were identified by flow cytometric means. $C D 3^{+} C D 4^{+} \mathrm{T}$ cells were further classified using different T cell markers (CD25, CD127, CD39, CD95). All cytometric measurements were performed using a standardized BD LSR-Fortessa platform. RA patients were compared according to their treatment with ABA, TCZ or RTX

Results: Eighteen out of 56 RA patients (32\%) received ABA, 25 patients (45\%) received $T C Z$ and 13 patients (23\%) were under CD20+ cell depletion therapy with RTX. RA patients receiving ABA displayed a significant decrease in $\mathrm{CD}^{+} \mathrm{CD} 4^{+} \mathrm{CD} 25^{+} \mathrm{CD} 127^{\mathrm{dim}}$ Tregs $(3.7 \% \pm 0.4)$ compared to patients with TCZ $(5.4 \% \pm 0.4, p=0.041)$ and patients under RTX treatment $(7.52 \% \pm 0.93, p=$ $0.026)$. CD $39^{+}$Tregs were significantly higher in RA patients treated with TCZ $(49.5 \%+3.2, p=0.000)$ or $\operatorname{RTX}(50.5 \% \pm 5.3, p=0.026)$ compared to patients receiving $\mathrm{ABA}(24.5 \% \pm 3.1)$. In addition, the frequency of $\mathrm{CD} 95^{+}$Tregs was significantly reduced in ABA patients compared to RTX patients $(59.6 \% \pm 3.1$ vs.76.7\% $\pm 3.6, p=0.014$; respectively). Interestingly, $T$ cells displaying an effector $\mathrm{T}$ cell phenotype $\left(\mathrm{CD}^{+} \mathrm{CD} 4^{+} \mathrm{CD} 25^{+/} \mathrm{CD} 127^{+}\right)$were increased in $\mathrm{ABA}$ treated patients compared to RTX treated patients $(59.6 \% \pm 3.1$ and $76.7 \% \pm 3.6, p=$ 0.002 ). Since none of our patients were a non-responder or had high disease activity, we could not analyse whether these changes are associated with treatment outcome.

Conclusion: Our data demonstrate that blockage of $\mathrm{T}$ cell stimulation via ABA leads to characteristic alterations in different regulatory and effector $T$ cells not seen in patients treated with TCZ or RTX. Further studies must clarify whether the analysis of regulatory and effector $T$ cell subpopulations before treatment initiation can be used as biomarker for treatment response.

References:

[1] Álvarez-Quiroga C, Abud-Mendoza C, Doníz-Padilla L, et al. CTLA-4-Ig therapy diminishes the frequency but enhances the function of treg cells in patients with rheumatoid arthritis. J Clin Immunol. 2011;31(4):588-595. doi: $10.1007 /$ s10875-011-9527-5

Acknowledgments: Work done in "CBmed" was funded by the Austrian Federal Government within the COMET K1 Centre Program, Land Steiermark and Land Wien.

Disclosure of Interests: None declared

DOI: 10.1136/annrheumdis-2020-eular.4894

\section{THU0032 MODIFIED PEPTIDES AS A NOVEL IMMUNOTHERAPY} FOR RHEUMATOID ARTHRITIS

E. Araklioti ${ }^{1,2}$, L. Herman ${ }^{1}$, N. Q. N. Nguyen ${ }^{1}$, R. Roohi Ahangarani ${ }^{1}$, M. Erak ${ }^{1}$, B. Lauwerys ${ }^{3}$, P. Durez ${ }^{3}$, V. Geenen ${ }^{2}$, A. Winkler ${ }^{4}$, M. Van Mechelen ${ }^{1}$, L. Vander Elst ${ }^{1}$, V. Carlier ${ }^{1} .{ }^{1}$ Imcyse SA, Liège, Belgium; ${ }^{2}$ University of Liège, GIGA,
Laboratory of Immunoendocrinology, Liège, Belgium; ${ }^{3}$ Cliniques Universitaires Saint-Luc, Department of Rheumatology, Brussels, Belgium; ${ }^{4}$ Pfizer Inc., Cambridge, United States of America

Background: Rheumatoid arthritis (RA) is a highly prevalent and severe systemic autoimmune disease associated with permanent disability and strong socio-economic burdens. Currently, there is no therapeutic treatment and RA patients rely on lifelong, costly treatments. Imcyse develops modified peptides eliciting antigen specific cytolytic CD4 T cells (cCD4+) that induce apoptosis of antigen presenting cells (APC) in a contact dependent manner. cCD4+ also induce apoptosis of autoantigen-specific bystander T-cells, activated by the same APC thus eliminating the risk of general immunosuppression. Peptides consist of $\mathrm{MHC}$ class II T cell epitopes of a target autoantigen modified in their flanking region by the addition of an amino acid sequence containing a thiol-disulphide oxidoreductase active motif ${ }^{1}$.

Objectives: The goal of this study was to synthesize modified peptides from a target RA autoantigen and test their potency to generate in vitro specific and cytolytic CD4+ T cells from RA patients.

Methods: We designed modified peptides from a target RA autoantigen after in silico and in vitro assessment to identify MHC II core binding region, HLA class II binding capacities and physiochemical properties.

CD4+ T cells were purified from PBMC of a newly diagnosed seropositive RA patient and co-cultured with autologous APC in the presence of the modified peptide. The CD4+ T cells were restimulated periodically. Peptide's ability to generate specific CD4+ T cells was evaluated by flow cytometric analysis of the expression of surface activation marker CD154 (CD40L). The peptide specific CD4+ T cell lines were sorted based on their surface CD154 expression. Their pro-apoptotic activity was assessed after overnight $(\mathrm{O} / \mathrm{N})$ co-culture of CD4+ T cells with fluorescent tracer labelled autologous lymphoblastoid cells lines (LCL). Flow cytometry quantification of LCL apoptosis was measured by annexin $\mathrm{V}$ staining. MHC II restriction of CD4+ T cells was demonstrated by the addition of blocking antibodies against HLA-DR, DP or DQ molecules.

Results: CD4+ T cells were in vitro expanded after six consecutive stimulations with the peptide. We investigated their specificity by flow cytometry and showed that $69 \%$ of $\mathrm{CD} 4+\mathrm{T}$ cells that were stimulated $\mathrm{O} / \mathrm{N}$ in the presence of the peptide expressed the activation marker CD154 versus $29 \%$ of CD4+ T cells that were stimulated in its absence. These cells were sorted based on CD154 expression following specific stimulation. Cell enrichment was then assessed by flow cytometric analysis. Data showed that more than $91 \%$ (background $3 \%$ ) were peptide specific based on CD154 expression.

After co-culture of CD4+ T cells with LCL, in independent experiments, Annexin $\mathrm{V}$ binding was detected on peptide loaded LCL, ranging from $69 \%$ to $89 \%$, while when LCL were kept unloaded these values were between $30 \%$ and $55 \%$, respectively, indicating that when specifically activated, these CD4+ T cells had pro-apoptotic activity. When both the peptide and blocking antibodies against HLA-DR, DP or DQ molecules added in the co-culture the pro-apoptotic activity was inhibited by $68 \%, 20 \%$ and $25 \%$, respectively.

Conclusion: The preliminary but very promising data show that our modified peptide generates peptide-specific CD4+ T cells with lytic properties that lyse target APC in an HLA class II specific manner. Our plan is to show that these CD4+ $T$ cells can also induce apoptosis in bystander $T$ cells and to further validate our results in additional RA donors.

\section{References:}

[1] Carlier, V. A., Vanderelst, L., Janssens, W. \& Jacquemin, M. G. Increased Synapse Formation Obtained by T Cell Epitopes Containing a CxxC Motif in Flanking Residues Convert CD4 + T Cells into Cytolytic Effectors. 7, (2012).

Disclosure of Interests: Eleni Araklioti Grant/research support from: This work was supported by Pfizer Inc. and by Walloon Region, Ludivine Herman Grant/ research support from: This work was supported by Pfizer Inc. and by Walloon Region, Ngoc Quynh Nhu Nguyen Grant/research support from: This work was supported by Pfizer Inc. and by Walloon Region, Roxana Roohi Ahangarani Grant/research support from: This work was supported by Pfizer Inc. and by WalIoon Region, Milos Erak Grant/research support from: This work was supported by Pfizer Inc. and by Walloon Region, Bernard Lauwerys: None declared, Patrick Durez Speakers bureau: AbbVie, Bristol-Myers Squibb, Celltrion, Eli Lilly, Pfizer, Sanofi, Vincent Geenen: None declared, Aaron Winkler Shareholder of: Shareholder of Pfizer, Inc, Employee of: Full time employee of Pfizer, Inc, Marcelle Van Mechelen Grant/research support from: This work was supported by Pfizer Inc. and by Walloon Region, Luc Vander Elst Grant/research support from: This work was supported by Pfizer Inc. and by Walloon Region, Vincent Carlier Grant/ research support from: This work was supported by Pfizer Inc. and by Walloon Region

DOI: 10.1136/annrheumdis-2020-eular-2714 